\title{
Design and Processing of a Novel Algorithm Using Anfis for New Generation of Cardiac Pacemakers
}

\author{
Asghar Dabiri Aghdam ${ }^{1}$, Nader Jafarnia Dabanloo ${ }^{1}$, Mohammad Sattari ${ }^{2}$, \\ Gholamreza Attarodi ${ }^{1}$, Nazanin Hemmati ${ }^{1}$ \\ ${ }^{1}$ Islamic Azad University, Science and Research Branch, Tehran \\ ${ }^{2}$ Islamic Azad University South Tehran Branch, Tehran
}

\begin{abstract}
This paper presents a new intelligent control algorithm using Anfis (Adaptive neuro fuzzy inference system) for new generation of Cardiac Pacemakers. Anfis uses both merits of Fuzzy and Neural networks (Learning and speed). Based on various states of body (rest, walking and exercising) and preprogramed states of patient (Sex, age, blood pressure) heart rate and amplitude of exciting pulses will be changed. A feedback from output ECG signal fed back and compared in Anfis controller. Anfis compared with other fuzzy control algorithms provides better control scheme for estimation and generation of pacing pulse parameters including rate and amplitude. Accelerometer sensors and HRV and GSR are used for stabilization of ECG signal during the three body states [1]. Old designs of pacemakers cannot solve problems but Fuzzy can do the job very fast and accurately. Anfis method designed externally regarding patient conditions and normal ECG signals data captured from a known database (based on age and sex of patient) then calculated in MATLAB (Simulink) and programmed directly or wirelessly by ultrasound signals (a novel proposed method). Previous researches [4-19-20] are based on fuzzy PID and fuzzy logic controller in patients with cardiovascular diseases. Anfis controller offers good adaptation of the heart rate and other ECG parameters to the physiological needs of patient.
\end{abstract}

\section{Introduction}

Anfis is a new fuzzy logic method for function approximation and control of miscellaneous engineering especially biomedical engineering. For example, AC and DC motor control and power plant control and even social engineering problems [10-11]. It is well known that pacemaker as one of the implantable cardiac devices for medical treatment of heart diseases has been widely used nowadays. It has become a therapeutic tool used worldwide with more than 250,000 pacemakers implants every year [2].

A pacemaker is a medical device that uses electrical impulses, delivered by electrodes contacting the heart muscles, to regulate the beating of the heart. Early diagnosis and medical treatment of heart diseases can prevent the sudden death of a patient. Thus, in order to provide rapid response, the first 10 -second is the most important part to show and compare the performance of the controllers [4]. An accelerometer placed in a pacemaker detects movement and patient's physical activity requiring an increased heart rate and generates an electronic signal that is proportional to physical activity [6]. QT interval rate response is physiologically accurate but slow to respond to the onset of moving. Consequently, to reduce these factors, two sensors are used to provide compensation, in that an ideal controller converges only when the system is stable. [3-5]

Due to the uncertainties contain imprecise information by combining the physiological demand, fuzzy logic controller realization for the pacemaker systems has been developed [7].In this paper, we present the advanced control for cardiac pacemaker using Adaptive neuro fuzzy inference system controller based on dual-sensors. This paper is organized as follows. Section II describes the rate regulation with dual-sensors in pacemaker systems using conventional PID and fuzzy PID control. Section III presents the ANFIS control design method for pacemaker systems. Section IV illustrates the various heart models. The tracking results in simulation are compared between our method and a PID fuzzy controller and FLC. Finally, at the last section conclusion will be presented.

\section{Conventional Control Scheme for Pacemaker Systems}

Conventional control and fuzzy PID controller has been demonstrated by Wei Vivien SHI [3-5]. The main idea of 
fuzzy control systems is to design a controller for a system that is structurally difficult to model due to naturally existing nonlinearities and other modeling complexities $[8,9]$. Fuzzy control system has the capability of transforming linguistic information and expert knowledge into control signals.

Block diagram of Shi's work is shown in fig. 1.

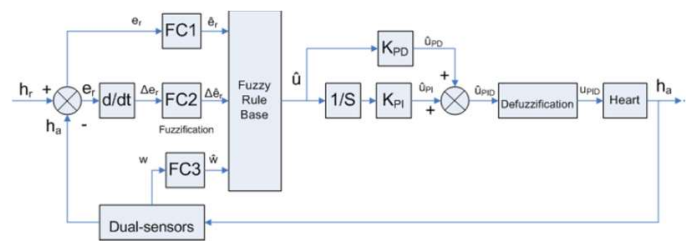

Figure 1. Design of fuzzy PID controller for dual sensors cardiac pacemaker system

\subsection{ANFIS Controller for Dual sensor Cardiac Pacemaker Systems}

A feedback from output ECG signal fed back and compared in fuzzy controller based on Anfis. Anfis compared with other fuzzy control algorithms provides better control scheme for estimation and generation of pacing pulse parameters including rate and amplitude.

\section{ANFIS Controller Design}

\subsection{ANFIS Concept}

ANN has strong learning capabilities at the numerical level. Fuzzy logic has a good capability of interpretability and can also integrate expert's knowledge. The

hybridization of both paradigms yields the capabilities of learning, good interpretation and incorporating prior knowledge. ANN can be used to learn the membership values for fuzzy systems, to construct IF-THEN rules, or to construct decision logic. The true scheme of the two paradigms is a hybrid neural/fuzzy system, which captures the merits of both the systems [12].

\subsection{The ANFIS Model}

The architecture of the ANFIS model is a graphical representation of the TS-FLC model. The general ANFIS control structure for the control of any plant is presented in this section [10], [11].

The functions of the various layers are given in the form of an algorithm as described below. The structure contains the same components as fis, except for the NN block. The network structure is composed of a set of units arranged into 5 interconnected network layers, L1 input variables, L2 input membership functions, L3 Fuzzy rules, L4 Output membership functions and L5 output values as shown in Fig.2. The ANFIS structure is tuned automatically by least-square-estimation and the backpropagation algorithm [12].

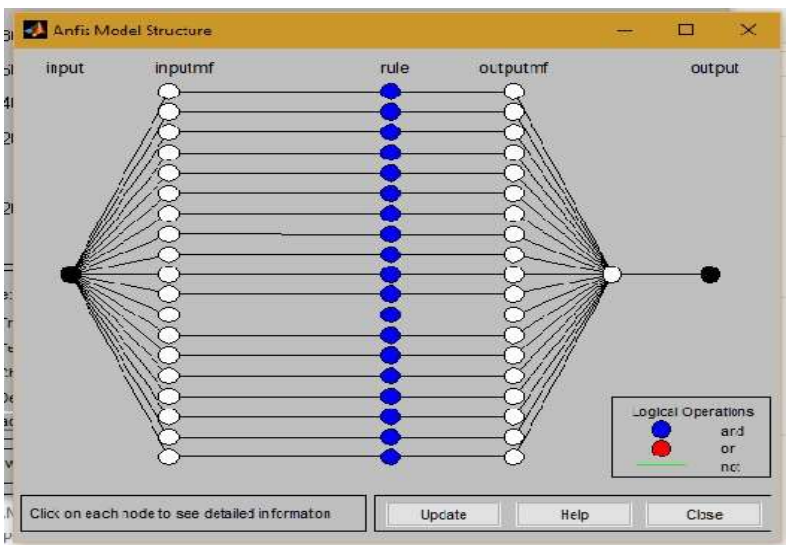

Fig. 2: ANFIS model with 1 input and 1 output showing the ANN architecture

\subsection{Simulation of Anfis controller in Simulink}

The simulation of designed controller shown in fig.3. first training data generated in a conventional PID heart controller (upper part). MATLAB PID feature used for optimization of step response of heart model then the in 1 and in3 signals exported to workspace of MATLAB(ver.14). Then these data entered as training data to the MATLAB ANFIS GUI toolbox (Anfisedit command). Load Data is used to input training data and Generate fis with Grid partitioning and hybrid back propagation and recursive least square as optimization method. The pacemaker model $8 /(\mathrm{s}+8)$ and the transfer function of the heart is chosen as given by the following: $169 /(\mathrm{s} 2+20.8 \mathrm{~s})$.

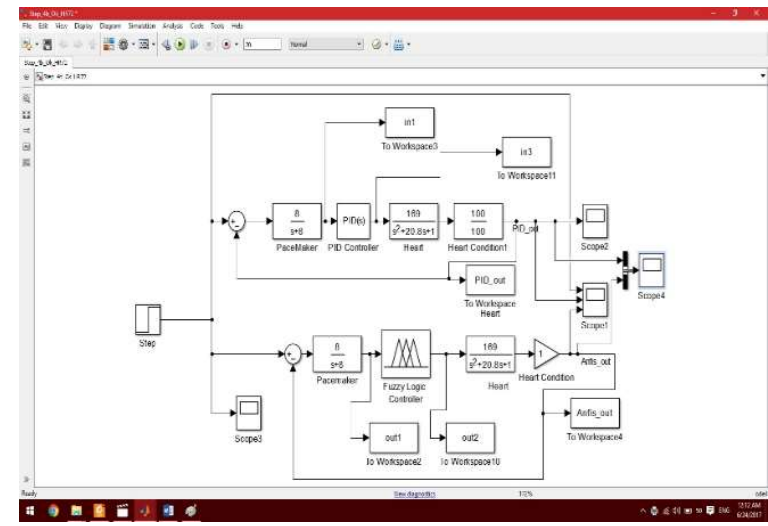

Fig. 3 Anfis and conventional PID simulation of Anfis controller using Simulink 
After the rule base is specified, the ANFIS adjusts only the MFs of the antecedents and the consequent parameters. The BP algorithm can be used to train both the premise and consequent parameters. Then pressing Train now starts design and optimization of input and output MF by hybrid methods described above. If we chose number of input MF's a large number $(>1000)$ it lasts very long time for MF's to be optimized (a couple of hours in a MSI laptop with Core i7 CPU and 16GByte of RAM) especially if we have more than two Input /Output.

After we got an acceptable error ( 0.38 at this research), we save the generated fis file and we can test it by using 'Test Now' button. Then the optimized fis file used in Fuzzy logic control box in Simulink. Figure 4 shows MATLAB PID tune toolbox output including Step response parameters (Rise time, Overshoot, Settling Time). The lower block in figure3 shows ANFIS controller with FLC box with fis file generated during Training phase of Anfisedit GUI toolbox.

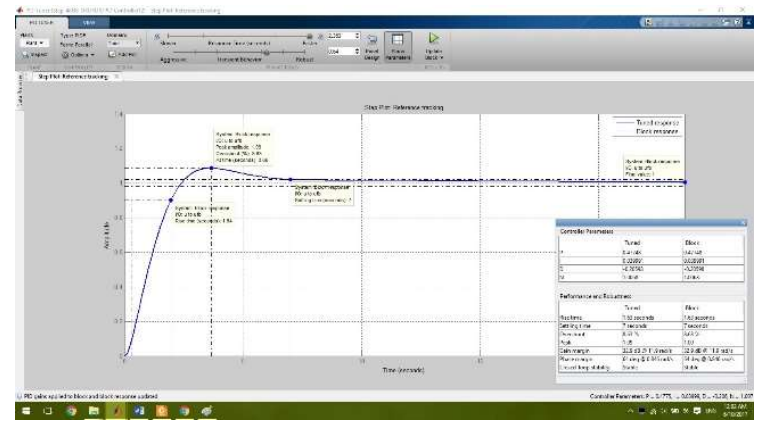

Fig.4 MATALB PID Auto tune toolbox

After running the SIMULINK model for 20 secs, the step input (72 bpm) and PID and ANFIS controller outputs and comparison between them, shown in Tables 1,2.

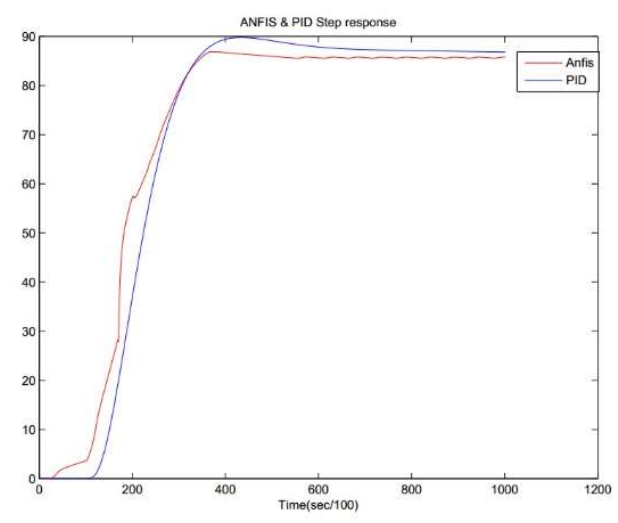

Fig. 5 Step response of conventional PID and ANFIS controller with $\mathrm{HR}=85 \mathrm{bpm}$

\subsection{Comparison of PID and fuzzy controller results}

Jyoti et al. compared a fuzzy controlled and a PID controller tuned with Ziegler-Nichols, Tyreus Luyben, and Relay methods; the work simulated and demonstrated that the fuzzy controller had a maximum overshoot less than all of the tuned PID controllers and improved rise time and settling time than at least two of the three tuning methods [21].

Table 1: Response parameter of ANFIS controller with various heart rates

\begin{tabular}{|c|c|c|c|}
\hline HR & $\begin{array}{c}\text { Rise } \\
\text { Time }\end{array}$ & $\begin{array}{l}\text { Settling Time } \\
\text { sec }\end{array}$ & $\begin{array}{c}\text { Max. } \\
\text { Overshoot }\end{array}$ \\
\hline 60 & 1.63 & 1.23 & 0.5 \\
\hline 72 & 1.97 & 1.39 & 1.53 \\
\hline 85 & 1.7 & 1.55 & 1.08 \\
\hline
\end{tabular}

Table 2: Comparison of result obtained by conventional and various PID and FPID and ANFIS controller

\begin{tabular}{|c|c|c|l|l|l|}
\hline Type & rmse & $\begin{array}{l}\text { Max. } \\
\text { error }\end{array}$ & $\begin{array}{l}\text { Max } \\
\text { Overshoot }\end{array}$ & $\begin{array}{l}\text { Rise } \\
\text { time(S) }\end{array}$ & $\begin{array}{l}\text { Settling } \\
\text { Time(s) }\end{array}$ \\
\hline FPID [4] & 0.889 & $1.72 \%$ & N. A & N. A & N. A \\
\hline Fuzzy [4] & 2.380 & $4.88 \%$ & N. A & N. A & N. A \\
\hline ANFIS & 0.14 & $1.4 \%$ & $1.08 \%$ & 1.7 & 1.55 \\
\hline PID & 0.16 & $2.03 \%$ & $5.85 \%$ & 1.43 & 9.88 \\
\hline REF [21] & N. A & N. A & $3.5 \%$ & 0.27 & 0.76 \\
\hline Fuzzy [22] & 1.034 & $4.88 \%$ & $\sim=0 \%$ & NA & NA \\
\hline
\end{tabular}

\section{Heart Model}

There are many heart models developed including mathematical [16-17-18-21] and electrical (Vanderpol and modified Vanderpol equations and YNI mathematical physiological model (Yanagihara, Noma, and Irisawa) [23]. within last 50 years. We have tried both IPFM [16] and Vanderpol Oscillators [13-15] and simulated using Simulink. Finally, we have selected transfer function model [21] in current work.

\section{Conclusion}

Heart Rate signals are used as a reliable indicator of heart diseases. These HR signals form the basis of functioning of a pacemaker. Pacemaker performance depends not only on sensors and the pacemaker circuitry but also on the performance of the controller. In the present work, different control techniques are analyzed to design Heart Rate controller. Initially a PID controller tuned with 
the help of MATLAB tune GUI tool to satisfy the different performance parameters. To improve the performance of the system, an intelligent ANFIS controller is designed with ANFIS toolbox , it is observed from the response of fuzzy controller that the parameters (maximum overshoot and RMSE and Maximum error) of ANFIS controller is better as compared to the conventional PID controller and other methods.

\section{Future Work}

Followings will be investigating in future works for ANFIS based design:

1. Van der Pol oscillator model will be added to SIMULINK simulations.

2. RMS error will be calculated.

3. Three body states will be simulated.

4. Using sensors (Accelerometers, HRV, GSR Minute ventilation, Blended Sensor... etc.).

\section{References}

[1] D. J. Woollons, "To beat or not to beat: the history and development of heart pacemakers," Engineering Science and Education Journal, vol. 4, 1995, pp.259-268.

[2] S. A. P. Haddad, R. P. M. Houben, and W. A. Serdijin, "The evolution of pacemakers," IEEE Engineering in Medicine and Biology Magazine, vol. 25, 2006, pp. 38-48.

[3] Shi, Wei Vivien. "Advanced Intelligent Control of Cardiac Pacemaker Systems Using a Fuzzy PID Controller." International Journal of Intelligent control and systems vol.18, No.2, June 2013,28-34

[4] Shi, W. (2013) A Novel Design of Fuzzy PID Controllers for Dual-Sensor Cardiac Pacemaker Systems. Open Journal of Applied Biosensor, 2, 29-38.

[5] W. V. Shi and M. C. Zhou, "Recent Advances of Sensors for Pacemakers," Proceeding of 2011 IEEE International Conference on Networking, Sensing and Control, Delft, 11-13 April 2011, pp. 520-525. doi:10.1109/ICNSC.2011.5874939

[6] P. L. Johnson, J. C. Newton, D. L. Rollins, W. M. Smith, and R. E. Ideker, "Adaptive pacing during ventricular fibrillation," Pacing and Clinical Electrophysiology, vol.26, 2003, pp.1824-1836

[7] P. Kligfield, K. G. Lax, and P. M. Okin, "QT interval-heart rate relation during exercise in normal men and women: Definition by linear regression analysis," Journal of the American College of Cardiology, vol. 28, 1996, pp. 1547-1555

[8] K. C. Sio and C. K. Lee, "Stability of Fuzzy PID Controllers," IEEE Transactions on Systems, Man and Cybernetics, Part A: Systems and Humans, Vol. 28, No. 4,1998, pp. 490-495. doi: $10.1109 / 3468.686710$

[9] L. X. Wang and J. M. Mendel, "Generating Fuzzy Rules by Learning from Examples," IEEE Transactions on Systems, Man and Cybernetics, Vol. 22, No. 6, 1992, pp. 1414-1427. doi:10.1109/21.199466

[10] Grabowski, P.Z. Kazmierkowski, M.P. Bose, B.K. Blaabjerg F, "A simple direct-torque neuro-fuzzy control of PWM-inverter-fed induction motor drive," IEEE Trans. on Industrial Electronics, Vol. 47, Issue 4, Aug. 2000, pp. 863-
870.

[11] Nasir Uddin M., Hao Wen, "Model Reference Adaptive Flux Observer Based Neuro-Fuzzy Controller for Induction Motor [12] Du K.-L. and M.N.S. Swamy, "Neural Networks in a Soft Computing Framework," Springer-

Verlag Pub. London, U.K.,

[13] A Mathematical Model of Automaticity in the Sinus Node and AV Junction Based on Weakly Coupled Relaxation Oscillators* C.R. KATHOLI, F. URTHALER,J. MACY, JR., ANDT.N. JAMES Department of Biomathematics, University of Alabama in Birmingham and the Cardiovascular Research and Training Center, University of Alabama School of Medicine, Birmingham, Alabama 32294 COMPUTERS AND BIOMEDICAL RESEARCH 1\&529543 (1977)

[14] An analysis of heart rhythm dynamics using a three-coupled oscillator model. Sandra R.F.S.M. Gois, Marcelo A. Savi *Universidade Federal do Rio de Janeiro, COPPEDepartment of Mechanical Engineering, P.O. Box 68503, 21941972 Rio de Janeiro, RJ, Brazil

[15] B.Z. Kaplan, I. Gabay, G. Sarafian, D. Sarafian, Biological applications of the "Filtered" Van der Pol oscillator, Journal of the Franklin Institute, Volume 345, Issue 3, 2008

[16] Krzysztof Grudziński, Jan J Żebrowski, Modelling cardiac pacemakers with relaxation oscillators, Physica A: Statistical Mechanics and its Applications, Volume 336, Issue 1, 2004, Pages 153-162

[17] McLernon D.C., N.J. Dabanloo, A. Ayatollahi, V.J. Majd, H.Zhang. A new nonlinear model for generating RR tachograms. Computer in Cardiology, Vol.31, 2004, pp.481484.

[18] Jafarnia-Dabanlooa N, McLernon DC, Zhang H, Ayatollahi

A, Johari-Majdd V. A modified Zeeman model for producing HRV signals and its application to ECG signal generation. Journal of Theoretical Biology 2007;244:180-189.

[19] M. Lak, N. Jafarnia Dabanloo and S. Kamaledin Setarehdan, "A chaotic model for generating heart rate variability signal using integral pulse frequency modulation," 2010 Computing in Cardiology, Belfast, 2010, pp. 857-858

[20] Shi, Wei Vivien and MengChu Zhou. "Fuzzy PID controllers for dual-sensor pacing systems in patients with bradycardias at rest." SMC (2011)

[21] Jyoti Yadav, Asha Rani and Girisha Garg. Article: Intelligent Heart Rate Controller For Cardiac Pacemaker. International Journal of Computer Applications, December

[22] Hamed, B. and Ras, A.A.K.A., 2015. Fuzzy Controller for Dual Sensors Cardiac Pacemaker System in Patients with Bradycardias at Rest. Intelligent Control and Automation, 6(03), p.159.

[23] J. Keener and J. Sneyd, Mathematical Physiology, New York: Springer; 1998

Address for correspondences.

Name. Asghar Dabiri Aghdam

Full postal address.: Islamic Azad University, Science and Research Branch, Tehran

Asghar.dabiri@srbiau.ac.ir 